P. K. Seidelmann

E. J. Santoro

K. F. Pulkkinen*

U. S. Naval Observatory

SUMMARY. This paper is a summary of the referenced complete paper which includes figures and references.

For the 1984 edition of the Astronomical Almanac and a number of other national ephemerides, a new set of ephemerides was introduced. These ephemerides were prepared cooperatively by the Jet Propulsion Laboratory and U. S. Naval Observatory using the observational data available, including radar and laser-ranging, spacecraft data, and optical data. They are primarily based on the 1976 IAU System of Astronomical Constants (IAU-Trans, 1977). The ephemerides are on the equator and dynamical equinox of J2000.0 as determined from the ephemerides themselves. The preparation of the ephemerides has revealed the need for clarification of certain definitions. In a number of cases, the past practice has been to utilize Newcomb's value or expression for a quantity as the definition of that quantity.

The new ephemerides are consistent with the accuracy of the observational data currently available. The ephemerides have indicated some discrepancies with the observational data which are due either to systematic discrepancies in the observational data, or inadequacies in our knowledge of a model of the solar system.

This paper is primarily concerned with the problem of making the theoretical computations of celestial mechanics agree with the observational data of the real world. While our real solar system moves in response to the actual masses and all the forces present, the theory we use to compute the ephemerides is limited to our present knowledge.

\title{
Observational Data
}

The observational data collected by Cohen, Hubbard and Oesterwinter were augmented by more recent observations of Pluto which were made by Barbieri et al (1979), by the Naval Observatory staff in Flagstaff and by Mulholland in Texas. The Flagstaff observations led to the discovery of the satellite of Pluto (Christy and Harrington 1978) which has

*now with OAO Corporation

99

J. Kovalevsky and V. A. Brumberg (eds.), Relativity in Celestial Mechanics and Astrometry, 99-103.

(C) 1986 by the IAU. 
drastically changed our knowledge of the mass of Pluto. The observations of Neptune have been collected and discussed by Jackson (1974) and augmented by more recent observations made with the Washington Transit Circle. For Jupiter, Saturn and Uranus the observational data taken in Washington have been augmented by observations from other observatories where systematic reduction to a common reference system has been possible. All of the observations have been given unit weight.

\section{Integration procedures}

The Jet Propulsion Laboratory has developed an extensive program for the integration of planetary and lunar ephemerides. They have incorporated optical observations, radar and laser ranging data and spacecraft observations for comparison with the ephemerides. The Naval Observatory has prepared an independent numerical integration scheme based on the integration of the orbital elements. Comparisons have been made between the two numerical integration methods to demonstrate the equivalence of the integrators. After fitting the integrations at the U. S. Naval Observatory to the extended outer planet observational data, normal points were formed for Uranus, Neptune and Pluto. The normal points were used for fitting the Jet Propulsion Laboratory integrations. Comparisons were then made between the various fits of the integrations to the observational data. The best prediction ephemeris which fit the available observational data has been used for the ephemerides now labeled DE200/LE200. Some modifications to the IAU (1976) system of astronomical constants have been necessary in order to achieve a best fit to the observational data. In particular the Viking satellite observational data required modifications to some of the outer planet masses.

\section{URANUS OBSERVATIONS}

When the new ephemerides were calculated, an attempt was made to fit all the observational data of Uranus. Unfortunately, the observational data indicated that the ephemerides were deviating systematically from the most recent observations by approximately $0 ! 5$. Therefore, the resulting ephemeris would be poor for predicting the positions of Uranus in the future. The one successful approach was to use only observational data after 1900. The use of the limited span of observational data provided what appears to be a satisfactory prediction ephemeris, but it does not explain the discrepancies from the pre-1900 observational data.

\section{NEPTUNE OBSERVATIONS}

This new ephemeris, like the previous Neptune ephemerides, is based on data for less than a complete orbital period. However, with one hundred thirty-five years of observational data out of the one hundred sixty year period of the planet, the ephemeris should be quite good. Unfortunately, based on the past experience, this probably will not be 
the case. In addition, there are pre-discovery observations of Neptune. Two observations were made by a person working for Lalande back in 1795. Those observations have residuals of approximately -12 " in right ascension. The residuals in declination are much smaller. In 1613 Galileo observed Neptune with respect to the Jupiter satellite system. The position of Neptune is indicated on an extension of a line, such that it is not definite whether the scale given for the drawing of the satellites of Jupiter also applies to the position of Neptune. Assuming that it does, the residuals of -32 " in right ascension and $40 "$ in declination indicate a large discrepancy between the observations and the ephemeris.

\section{SATURN OBSERVATIONS}

The declination observations of Saturn indicate a systematic difference which depends on the observatory making the observations. The systematic difference is consistent with observations of one limb of the planet corrected to the apparent center of Saturn based on an inaccurate apparent flattening of Saturn. This discrepancy has a periodic nature, which is exactly the period of Saturn. Thus, these observations would give a different orbital plane for Saturn and the inclination and longitude of the node would be systematically affected.

\section{SOLAR OBSERVATIONS}

Since a one second per century correction was introduced for the constant of precession and for the motion of the equinox, there is concern about the possibility of a one second per century discrepancy in the ephemerides. Particularly, the concern centers around the motion of the Sun, which will be discussed later. Search for this possible discrepancy led us to look at normal points of the solar observations plotted as a function of the seasons. The residuals in $r$ ight ascension for spring and fall show little systematic trend. The right ascension residuals for summer and winter show the possibility of a systematic trend. The declination residuals for spring and fall show a possible trend, but the summer and winter normal point residuals in declination show a definite trend.

There are a number of possible sources of seasonally dependent systematic discrepancies between the solar ephemeris and the observations; e.g. refraction, day/night corrections, telescope heating, and catalog clock star errors. Thus there are a number of conceivable explanations for the existence of a systematic discrepancy in the observations themselves.

\section{CONSTANTS OF NEW EPHEMERIDES}

The new ephemerides have been fit to all the available observational data subject to some limitations. This has revealed the 
need for an improved definition of the dynamical equinox and the obliquity. The ephemerides have been used to determine their own equinox. While it would be possible to try to put the ephemerides on the FK5 equinox, at some level of accuracy that attempt would fail and the ephemerides would be on neither the dynamical equinox of the ephemerides, nor the catalog equinox of the FK5. Therefore, it was judged to be preferable to put the ephemerides on their own equinox. Similiarly, there is no way to force a given value of obliquity into the ephemeris of the Earth and solve for the obliquity from the observations, so the observational data determined the orbital plane of the Earth.

\section{IS THERE A ONE SECOND PER CENTURY DISCREPANCY?}

Stumpff and Lieske (1984) have inferred the existence of a one second per century error in one of the following: (1) The new constant of precession could be wrong by one second per century. (2) The inertial reference system of the new ephemerides could contain a one second per century rotation. (3) The classical equation of Newcomb for the solar longitude of the equinox of date could be wrong by one second per century.

Equinox motion means that the error in the equinox of the FK4 varies as a function of time. Thus, it would appear that the tropical year and the expression for solar longitude for the equinox of date, while independent of the constant of precession, may be dependent on a correction for equinox motion. It should be pointed out that some of the stellar data underlying the FK4 and its proper motions are from the same catalogs that form the basis for Newcomb's expression for the longitude of the Sun. The solar data was used to determine the equinox for the stellar data. Therefore, if we accept the time dependent correction to the FK4 equinox, then we must accept the fact that Newcomb's expression for the longitude of the Sun for the equinox of date may also require a correction. If this is the case, it would explain the one second per century discrepancy given by Stumpff and Lieske.

An alternative argument put forth by Fricke (1982) is that the time dependent equinox correction is due to a magnitude effect in the star catalogs and is independent of Newcomb's expression for the longitude of the Sun. If that is true then either the new precession constant is wrong by one second per century, or the inertial reference system of the ephemerides rotates at one second per century. These possibilities will require further investigation.

\section{REFERENCE}

Seidelmann, P. K., Santoro, E. J. and Pulkkinen, K. F. 1985 'Systematic Differences between Planetary Observations and Ephemerides' in Dynamical Astronomy edited by V. Szebehely and B. Balazs, University of Texas Press, Austin, Texas, P. 55. 


\section{DISCUSSION}

Branham : did you consider subsets of the XIX-th century observations which, according to you, caused some of the problems in the reduction ? My experience with XIX-th century observations of minor planets is that they are generally acceptable, with the exception of mural circle observations.

Seidelmann : we have studied the observations from each observatory 1ooking for systematic deviations as a function of observatory. We studied an individual observatory in detail, rereducing each night's observations based on the star positions. This improved the scatter of the observations, but did not cause a systematic change. Thus, we have not found a source of the systematic trends in the observations. It should be pointed out that the XIX-th century observations are not necessarily systematically in error. We have fit the XIX-th century observations because we need a prediction ephemeris for the future.

A comparison between ephemerides and observations in right ascension for the Sun, Mercury and Venus was made for two cases, one without equinox motion and the other with the equinox motion value of FK4 with the FK5 correction. The comparison in the first case showed a secular rate of differences. In the second case, the solar residuals do not indicate a secular trend, but the Mercury and Venus residuals do indicate a secular trend. The source of this trend is, at this time, uncertain.

Lederle : have you tried to solve for the equinox and the equinox motion from this data?

Seidelmann : no. We are still seeking other possible sources for these discrepancies. The observations were used for equinox solutions as part of the individual observational catalogs.

Lieske : from your suggestion that a $1^{\prime \prime}$ drift exists due to equinox motion, is it correct to suggest (using the terminology of the paper in A. and A. by Dr Stumpff and me) that the "discrepancy" is due to an erroneous longitude adopted by Newcomb?

Seidelmann : I think that when you correct the constant of precession, you must also introduce the correction for the motion of the equinox. The equinox of date for Newcomb's theory was based on observational data that also determined the equinox of the star catalogues. Newcomb's expression for the longitude may have been correct in that reference system, but it must be properly modified for the FK5 reference equinox of J 2000.0. 